\title{
TRANSPIRING COOLING OF A SCRAM-JET ENGINE COMBUSTION CHAMBER
}

\author{
Sang H. Choi ${ }^{\dagger}$, Stephen J. Scotti ${ }^{\dagger}$, Kyo D. Song ${ }^{\ddagger}$, and Heidi Ries ${ }^{\ddagger}$ \\ NASA Langley Research Center \\ Hampton, Virginia 23681-0001
}

\begin{abstract}
The peak cold-wall heating rate generated in a combustion chamber of a scram-jet engine can exceed $2000 \mathrm{Btu} / \mathrm{ft}^{2} \mathrm{sec}\left(\sim 2344 \mathrm{~W} / \mathrm{cm}^{2}\right)$. Therefore, a very effective heat dissipation mechanism is required to sustain such a high heating load. This research focused on the transpiration cooling mechanism that appears to be a promising approach to remove a large amount of heat from the engine wall. The transpiration cooling mechanism has two aspects. First, initial computations suggest that there is a reduction, as much as $75 \%$, in the heat flux incident on the combustion chamber wall due to the transpirant modifying the combustor boundary layer. Secondly, the heat reaching the combustor wall is removed from the structure in a very effective manner by the transpirant. It is the second of these two mechanisms that is investigated experimentally in the subject paper.
\end{abstract}

A transpiration cooling experiment using a radiant heating method, that provided a heat flux as high as $200 \mathrm{Btu} / \mathrm{ft}^{2} \mathrm{sec}\left(\sim 234 \mathrm{~W} / \mathrm{cm}^{2}\right)$ on the surface of a specimen, was performed. The experiment utilized an arc-lamp facility $(60-\mathrm{kW}$ radiant power output) to provide a uniform heat flux to a test specimen. For safety reasons, helium gas was used as the transpirant in the experiments. The specimens were $1.9-\mathrm{cm}$ diameter sintered, powdered-stainless-steel tubes of various porosities and a $2.54-\mathrm{cm}$ square tube with perforated multi-layered walls. A $15-\mathrm{cm}$ portion of each specimen was heated. The cooling effectivenesses and efficiencies by transpiration for each specimen were obtained using the experimental results. During the testing, various test specimens displayed a choking phenomenon in which the transpirant flow was limited as the heat flux was increased. The paper includes a preliminary analysis of the transpiration cooling mechanism and a scaling conversion study that translates the results from helium tests into the case when a hydrogen medium is used.

\footnotetext{
† Research Engineers with NASA Langley Research Center. † Professors, Norfolk State University, Norfolk, Virginia. Copyright (C) 1997 by the American Institute of Aeronautics and Astronautics, Inc. All rights reserved.
}

\section{INTRODUCTION}

High heating rates sustained by a hypersonic vehicle, as shown in Fig. 1, are a significant problem in the development of such vehicles, with the leading edges and scram-jet combustion chamber of such vehicles subject to very high heating loads. Fig. 2 shows estimated heating loads within a scram-jet engine at a Mach 15 flight condition. The most extreme heat loads are created within the engine combustor, and these conditions are the most difficult to accommodate in the thermal-structural design of the engine. The passive, ablative thermal protection systems that have been studied for many years for single-use reentry vehicles are not appropriate for this application. The thermal protection system for a scram-jet engine must differ from reentry vehicles, requiring considerations of reusability, maintenance of precise geometric contours, and incorporation of a hydrogen propellant.

Generally, heat dissipation mechanisms adopted for such heating loads as shown in Fig. 2, are the employment of ablative materials, regenerative cooling using hydrogen fuel, gas film or transpiration cooling, or active heat-pipe cooling. The ablative heat dissipation mechanism appears inapplicable for continuous operation of hypersonic vehicles because of significant ablative loss of combustor wall materials. The use of regenerative cooling can be shown to induce large efficiency penalties in the hypersonic vehicle because high fuel pressures and excessive fuel are needed to reduce the combustor wall temperature to acceptable values. However, the gas bleeding or transpiration cooling mechanism appears attractive since the bleed medium passing through the pores of the wall panels removes the absorbed heat, and also effectively restructures the boundary layer, which may reduce heating of the combustor wall. The restructured boundary layer may alleviate convective heating by shielding the wall from hot combustor gases. If the transpirant adopted for cooling is a propellant (such as hydrogen), the injection of propellant into the scram-jet combustion chamber through the combustor wall may be beneficial for better mixing of propellant in a supersonic combustion process, but it may also increase heating of the combustor wall due to the local burning near the wall. 
In a scram-jet, a tremendous heat flux (average $>500 \mathrm{Btu} / \mathrm{ft}^{2} \cdot \mathrm{sec}$ or $>584 \mathrm{~W} / \mathrm{cm}^{2}$ ) is generated from the supersonic combustion of hydrogen fuel. As a result, the engine wall of scram-jet is exposed to a high heating load. To alleviate such a high heating load, the transpiration cooling mechanism was investigated experimentally to determine its cooling effectiveness and its capability to shield the combustor wall from hot combustor gases. Only the former experimental investigation is described in the present paper. Thus, a radiative heating technique that avoids the uncertainty of the incident heat flux caused by transpiration-boundary layer interactions was used in the experimental investigation. The experimental specimens and procedures are described subsequently. The cooling effectivenesses of the transpiration specimens are then determined from measured outer and inner wall temperatures. The experimental results, obtained using a helium test medium, are then scaled to operation using a hydrogen fuel.

\section{EXPERIMENT}

The setup for transpiration cooling experiment (TCE) is shown Fig. 3. The test bench for the TCE consists of an arc-lamp, an elliptical reflector cavity, a test section at where a specimen is placed for exposure to intense light, three pressure gages, two thermocouples, an infrared thermometer, and two flow-meters as shown in the figure. A solar simulator arc-lamp was the heating source for the TCE. The argon arc-lamp generates an emission spectra at a $6,500 \mathrm{~K}$ color temperature. The emission of the arc is similar to a solar spectrum as shown in Fig. 4. The arc-tube is a cylindrical volume $15-\mathrm{cm}$ long and $1.1-\mathrm{cm}$ in diameter. The system generates a $60-\mathrm{kW}$ optical power output from a $150-\mathrm{kW}$ electrical power input. The radiant emission from arc-lamp is focused on a specimen to simulate the heat reaching the combustor wall through a transpiration-altered combustor boundary layer. To effectively collect and concentrate optical power from the arc lamp on the specimen, an elliptical reflector conduit is used as shown in Fig. 5. The arc lamp is placed at a focal line of the elliptical reflector conduit and the specimen at the other focal line. Once a specimen is placed on a focal line, the reflective side-plates seals the elliptical reflector conduit to contain optical beam energy within the cavity.

A scram-jet is designed to use the cryogenic hydrogen as a propellant and also as a coolant in its regenerative cycle. In the experimental study, room temperature helium gas was used in place of cryogenic liquid hydrogen $\left(L H_{2}\right)$ for safety reasons. Hence, a compensatory adjustment for heating load was made for the use of room tempera- ture helium in which the matched parameters were maximum transpirant temperature and mass flowrate. The energy required for heating cryogenic hydrogen up to $1,000 \mathrm{~K}$ is $12,127 \cdot m_{H_{2}}$ in Joule, but $4,774 \cdot m_{\mathrm{He}}$ in Joule for room temperature helium where the mass flows, $m_{H_{2}}$ and $m_{\mathrm{He}}$, are in grams. For the same mass flow rate, the experiment with room temperature helium would require a heat flux level of just only $200 \mathrm{Btu} / \mathrm{ft}^{2} \cdot \mathrm{sec}\left(234 \mathrm{~W} / \mathrm{cm}^{2}\right)$ in place of the average heat flux, $500 \mathrm{Btu} / \mathrm{ft}^{2} \cdot \sec \left(584 \mathrm{~W} / \mathrm{cm}^{2}\right)$, required for cryogenic hydrogen. The maximum experimental heat flux is determined by the lamp power setting and the size of a specimen. Using the maximum optical power $(\sim 60 \mathrm{~kW})$ of the arc lamp for a specimen with $2 \mathrm{~cm}$ in diameter and $15 \mathrm{~cm}$ in length, the irradiance on the specimen surface could reach approximately $627 \mathrm{~W} / \mathrm{cm}^{2}$ which is more than enough for desired experiment. A specimen is subjected to a heat flux that can be adjusted from a few tens of $B t u / f t^{2} \cdot s e c$ to maximum $200 \mathrm{Btu} / \mathrm{ft}^{2} \cdot \mathrm{sec}\left(234 \mathrm{~W} / \mathrm{cm}^{2}\right)$. The specimens in these experiments were limited, by power setting and transpiration flow control, to a peak temperature less than $1,000 \mathrm{~K}$.

Specimens prepared for test were of two kinds: the one being stainless steel tubes which have a 15 $\mathrm{cm}$ long middle portion of porous, sintered powdered stainless steel [1] and the other a square cross-section tube $(2.54 \mathrm{~cm}$ by $2.54 \mathrm{~cm}$ and 15 $\mathrm{cm}$ long) which was fabricated with multi-layers of thin and perforated Inconel plates [2]. All specimens have two thermocouples imbedded at both the inside and outside surfaces of their middle portion to measure the surface temperature. The thermocouple wires run through the inside of tube. An additional thermocouple was used to measure gas temperature. A thermal radiometer was also used for measuring the outside temperatures of the specimen, but it was found that the measurements were not reliable because of the changing emissivity of the specimens during the tests. In general, the thermo-physical characteristics of porous specimens were determined by a thermal imaging system (TIS), thermocouples, flowmeters with controller, pressure transducers, and anemometer for monitoring the outside gas flow conditions. Two different ranges of flowmeters were used for measuring flow-rates. A Fluke data acquisition system was used for logging the measured data of temperature and the flow-rates.

The experimental routine was set up for defining a correlation of the helium flow-rate versus surface temperature of porous specimens. In one mode of operation, once the probes and system were prepared, helium gas was flowed into a specimen and the flow rate allowed to stabilize before any attempts of heating. Then, the arc-lamp was 
turned on and its beam power was step-by-step increased from a starting point to a final power level to see the responsiveness of heated materials under a constant flow of helium gas. In other cases, the beam power was set to a constant level while the gas flow-rate was varied.

\section{RESULTS AND DISCUSSION}

The first specimens used in the experiment were three porous stainless steel tubes $(1.5,3$, and 5 micron average pore sizes). The performance of a porous conduit with the square cross-section was also investigated. The porous wall was built with lamination of thin and perforated Inconel plates (so-called Lamilloy [2]). When helium gas was continuously charged into a specimen, it flowed through the porous wall of a specimen while the inner and outer surface temperatures of the specimen were monitored in time. The pressure and flow-rates of helium and the heat flux applied (calculated from power settings) were also monitored. Fig. 6 shows the surface temperatures of the threemicron specimen [1] that was measured by the infrared thermometer and thermocouples. The results are plotted as a function of medium flowrates.

With a quarter of the arc-lamp maximum power $(60 \mathrm{~kW})$, the outside surface temperature was elevated to $1,100 \mathrm{C}(1,373 \mathrm{~K})$ within two minutes without helium flow. The outside surface temperature decreased as helium gas started flowing and its flow-rate was increased. However, the inside wall temperature of the specimen dropped more rapidly as the flow-rates increased, as shown in Fig. 7. The inner surface temperatures were much lower than the outer surface temperatures since the incoming gas medium was maintained at room temperature and was quite a bit cooler than the gas blowing out through porous wall. The gas medium that goes through a porous wall is heated up drastically and becomes less effective for cooling when it reaches the outside surface than at the inside surface. Hence, the outside surface temperature drops gradually even though the flow-rate increases. Such a contrasting picture is shown in Fig. 8. The effectiveness of the transpiration cooling may be defined as

$$
\eta=\frac{T_{g-o u t}-T_{g-i n}}{T_{s}-T_{g-i n}}
$$

where $\eta$ is the effectiveness of the transpiration, $T_{g-i n}$ and $T_{g-o u t}$ are the gas temperatures before and after passing through the porous wall, and $T_{s}$ is the surface temperature of the porous wall on which an intense heat flux is incident. In experiment, the measurement of actual gas temperatures after passing through the specimen's porous wall couldn't be made because the gas and the measurement probe were all exposed to an intense optical radiation. The temperatures measured were the ones from a thermocouple attached to the outside surface of a specimen and a thermocouple placed inside the tube specimen. Therefore, the difference in gas temperatures was estimated by using a heat flux and flow-rate of medium as such $T_{g-\text { out }}-T_{g-\text { in }} \simeq \frac{\dot{Q}^{\prime \prime}}{m C_{p}}$. The cooling effectiveness by transpiration of helium gas was obtained by the following approximation for the relative.

$$
\eta \simeq \frac{\dot{Q}^{\prime \prime}}{\dot{m} C_{p}\left(T_{s}-T_{g-i n}\right)}
$$

where, $\dot{Q}^{\prime \prime}$ is the heat flux, $\dot{m}$ is the mass flow-rate through the transpiration and $C_{p}$ is the specific heat of medium.

The cooling effectivenesses for the 3-micron porous and square cross-section specimens are plotted along with helium flow-rates (Fig. 9). The results show that the cooling effectiveness is decreased along with the flow-rates. However, Fig. 9 shows that for 3-micron porous specimens the decrease in cooling effectiveness along with the flow-rates of helium coolant is steeper at a high heating load than those at low heating loads. The square crosssection specimen shows a slow decrease in transpiration cooling effectiveness as compared to the results of 3-micron porous specimens along with flow-rates. And the square cross-section specimen under the heat flux of $40 \mathrm{Btu} / \mathrm{ft}^{2} \cdot \sec (46.7$ $W / \mathrm{cm}^{2}$ ) also demonstrates better cooling effectivenesses than for a 3 -micron porous specimen at 45 $B t u / f t^{2} \cdot \sec \left(52.6 \mathrm{~W} / \mathrm{cm}^{2}\right)$.

The perforated holes on a thin layer undergo a relatively small change in their sizes or open-hole diameter due to the one-dimensional thermal expansion and let a transpirant flow without much alleviation. However, the sintered wall of 3 -micron porous specimens undergoes a three dimensional thermal expansion that reduces the cross-section area of flow passage and eventually attributes to a slow flow of transpirant by choking. When a $200 \mathrm{Btu} / \mathrm{ft}^{2} \cdot \sec \left(234 \mathrm{~W} / \mathrm{cm}^{2}\right)$ heat flux (300 amperes of the arc-lamp system) was applied, the medium flow through the 3-micron porous specimen was completely choked due to the thermal expansion of the sintered material that eventually blocked the passage of medium. This phenomenon repeatedly appeared on high heat flux experiments that were carried out with the same test conditions. After chocking, the pressures ( $\sim 40$ si or $0.28 \mathrm{MPa})$ measured at the flowmeters located at upstream and downstream were identical. A history of surface 
temperature with a uniform flow-rates is shown in Fig. 10. The temperature was rapidly stabilized at $850^{\circ} \mathrm{C}$, and was very responsive to the flow-rates changes as seen by the quick response of surface temperature with the flow perturbation at 55 seconds. A time history of the surface temperatures was plotted with various heat fluxes from 66 to $100 \mathrm{Btu} / \mathrm{ft}^{2} \cdot \sec \left(77\right.$ to $\left.117 \mathrm{~W} / \mathrm{cm}^{2}\right)$ at a constant 60 SLPM flow-rate in Fig. 11. The rapid response of the specimen to the changes in heating level is again seen in this figure. Fig. 12 shows the surface temperature of the porous conduit specimen having the square cross-section with respect to flow-rates under various heat fluxes. This figure also shows the temperatures measured by the IR camera. For instance, at the heat flux of 53 $B t u / \mathrm{ft}^{2} \cdot s\left(62 \mathrm{~W} / \mathrm{cm}^{2}\right)$ there are a significant difference in temperatures that were measured by either thermocouples and IR camera. The temperature profiles do not show equilibrium temperatures corresponding to heat fluxes and medium flow-rates. However, under the heat fluxes of $105\left(123 \mathrm{~W} / \mathrm{cm}^{2}\right)$ and $130 \mathrm{Btu} / \mathrm{ft}^{2} \cdot \mathrm{sec}\left(152 \mathrm{~W} / \mathrm{cm}^{2}\right)$ and flow-rates between 400 and 500 SLPM, the surface temperature profiles show a tendency that would reach at an equilibrium temperature if the flow-rates were continued to increase. Since the maximum flowrates of helium was limited to 500 SLPM in the experiments, the profiles in Fig. 12 could not show the equilibrium points. In a practical sense the results of equilibrium tendency under the heat fluxes of $105\left(123 \mathrm{~W} / \mathrm{cm}^{2}\right)$ and $130 \mathrm{Btu} / \mathrm{ft} \mathrm{t}^{2} \cdot \mathrm{sec}(152$ $W / \mathrm{cm}^{2}$ ) are obscure and it is difficult to explain what causes these results with limited number of tests.

It is important to show the performance in an actual scram-jet application when hydrogen is used as the transpirant instead of helium. The conversion of the test data was based on the test parameters of helium transpirant and the scaling factors of thermo-physical properties of hydrogen as opposed to those of helium. The Darcy equation for a flow of gas through a porous media is described as

$$
V=-C_{1} \frac{\kappa}{\mu} \frac{\left(P^{2}-P_{a}^{2}\right)}{2 P t}, \quad \mathrm{~cm} / \mathrm{sec}
$$

where $C_{1}$ is a constant $\left(1.333 \times 10^{4}\right), \kappa$ is the permeability of the material $\left(\mathrm{m}^{2}\right), \mu$ the viscosity $(\mathrm{kg} / \mathrm{m} \cdot \mathrm{sec}), t$ the thickness $(\mathrm{cm})$ of the porous material, $P$ and $P_{a}$ the pressures (torr) inside and outside the porous media, respectively. Nominal values for the specific heat, permeability $(\kappa)$, and viscosity $(\mu)$ are listed in Table I. Using the equation, the velocities of the helium and hydrogen are calculated and plotted in Fig. 13 along with measured data derived from the flowmeter. The calculated results for helium qualitatively agree with the measured results, and show that hydrogen would have appreciably higher velocities for the same differential pressures.

The thermo-physical properties of gases through a porous medium, such as thermal conductivity, specific heat, viscosity, and diffusivity, in terms of the gas temperatures were evaluated with the data that was available in the heat transfer handbook $[3]$ and the results from the experimental data. The thermal conductivities of $\mathrm{He}$ and $\mathrm{H}_{2}$ were quite linear with gas temperature from 300 to $1500 \mathrm{~K}$ (Fig. 14). Fig. 15 shows that the differential pressures of the helium and hydrogen gases are increased with the gas flow-rates.

Table I. Parameters of Gaseous Transpirants

\begin{tabular}{|l|l|l|l|}
\hline Gas & $C_{p}(\mathrm{cal} / \mathrm{g} \mathrm{C})$ & $\mu\left(\mathrm{m}^{2} /\right.$ Darcy $)$ & $\kappa$ (Darcy) \\
\hline $\mathrm{He}$ & 1.25 & $471.3 \times 10^{-7}$ & 0.001 \\
\hline$H_{2}$ & 4.08 & $213.7 \times 10^{-7}$ & 0.001 \\
\hline
\end{tabular}

For a porous specimen the heat balance can be formulated as

$$
Q_{i n}=Q_{c o n v}+Q_{r a d}+Q_{c o n d}
$$

where, $Q_{i n}$ is the input power, $Q_{\text {cond }}$ the conduction loss, $Q_{\text {conv }}$ the convective loss, and $Q_{\text {rad }}$ the radiative loss. The density of the gas changes with temperature and is obtained from the state equation of gas, $P=\rho R T$. Let $M$ denote the atomic weight of a gas in $g /$ mole, then by introducing suitable conversion factors, the density of the gas as a function of the temperature can be written as

$$
\rho=\frac{0.101325 \mathrm{M}}{R T} \quad\left(\mathrm{~g} / \mathrm{cm}^{3}\right)
$$

Densities of helium and hydrogen at room temperature can be calculated as $\rho_{\mathrm{He}}=0.1785 \times 10^{-3}$ and $\rho_{\mathrm{H}_{2}}=0.8928 \times 10^{-4}\left(\mathrm{~g} / \mathrm{cm}^{3}\right)$. Therefore, the heat loss by convection, $Q_{\text {conv }}$, can be written as

$$
Q_{c o n v}=C_{2} \cdot A_{s} C_{p} V \rho \Delta T
$$

where $C_{2}$ is a conversion constant (4190) and $A_{s}$ is the exposure area of a specimen. Using the above equation, the $Q_{c o n v}$ versus the flow-rates is plotted as shown in Fig. 16. This result indicates that the transpiration cooling effects by hydrogen gas far exceed than those by helium. Now consider that the cryogenic hydrogen is used for transpiration cooling. In this case the incoming temperature of medium is very low that it can allow, relatively, a very high heat flux while maintaining the surface temperature at a desired level. 
The performance comparison between a sintered circular tube and a multi-layered and multi-perforated square cross-section tube can be seen from Figs. 17 and 18. To maintain the surface temperature around $600^{\circ} \mathrm{C}$, the flow-rates required for a sintered tube element was 75 SLPM after a projected run (750 seconds). For a multi-layered and multiperforated square cross-section tube, a 500 SLPM flow-rates was required. As mentioned earlier, this fact reflects the large difference in throughthe-thickness thermal conductivities, the effective porosity differences, pore size, material difference between these two specimens tested under a similar condition setting. Strangely, we see some irregularities on temperature profiles for the inside and outside surfaces of a sintered tube specimen. For some cases, the inside temperatures were higher than the outside temperatures. Following the temperature profiles closely, one may find that the spikes distinctively respond to the flow-rates. This is probably due to the fact that two different types of temperature probes, the one by a thermocouple and the other for the outside surface by an infrared camera, were used. The IR camera uses a spectral band between $3.5 \mu \mathrm{m}$ and $4.5 \mu \mathrm{m}$, where there exist virtually no emission spectra from the argon arc cavity, to determine the emissive power from a specimen. Often the optical characteristics of specimen surface were changed during a heating period while the emissivity setting of IR camera was fixed. Hence, the temperature differences between measured by the IR camera and thermocouple over a wide range of temperature clearly exist.

\section{CONCLUSIONS}

The one of the distinctive effects observed through the experiments was the choking effect by thermal expansion that blocks the flow passage of transpirant through a porous wall. The choking effect was so significant for the 3-micron sintered specimens that the overall flow was terminated at one point when a $200 \mathrm{Btu} / \mathrm{ft}^{2} \cdot \sec \left(234 \mathrm{~W} / \mathrm{cm}^{2}\right)$ heat flux was applied. Such an effect was not noticed with the square cross-section specimen, which was fabricated with a multi-layered wall with multiperforated holes, at any heat flux levels. The perforated holes on each thin layer of the multi-layered wall are only affected by a 1-dimensional thermal expansion while heated. On the other hand, the pore structure of sintered specimens were affected by the three dimensional thermal expansion of sintered grains. Hence, the porous passages within sintered specimens became shrunken and eventually sealed off when an extensive heat was loaded. The through-the-thickness thermal conductivity of the square cross-section specimens is much lower than that of the sintered materials because of the gap-spacing between layers. The low thermal conductivity slows down the heating of inner layers. Thus, the transpirant that passes through a layer to layer is not heated up as much as the case of sintered materials prior to reaching the outer layer surface. Hence, the transpiration cooling effectiveness for the inside wall of the square cross-section specimens is much better than that of sintered ones. For the squared specimen, the cooling effectiveness of the inner surface was increased from $20 \%$ to $75 \%$ while the cooling effectiveness of the outside surface is constant as about $20 \%$ when the flow-rate is changed from 20 to 80 SLPM. However, the cooling effectiveness for the outside wall is not as effective as that of a sintered tube because of its poor through-the-thickness thermal conductivity. The heat is mostly accumulated at the top layer of the wall and increases the surface temperature much higher than that of sintered one. Even though a large amount of transpirant passes through, the cooling effect on the top layer is limited by heat accumulation on the outer layer. However, it is still envisioned that the multi-layered wall with multi-perforated structure is better than a sintered structure because at high temperature it maintains its structural integrity and shows no clogging phenomenon.

The results of a scaling conversion study for the case when hydrogen would be used in place of helium show much higher cooling effects since hydrogen has higher thermal conductivity, higher specific heat, and higher passing velocity due to small atomic cross-section and a low viscosity than helium. In real applications where a cryogenic liquid hydrogen is used, the highly efficient cooling by hydrogen transpirant would allow much higher heat flux handling capability than defined with helium in this experiment.

\section{ACKNOWLEDGEMENTS}

This work was partially supported by the NASA Langley Research Center Grant (Grant NAG-11513).

\section{REFERENCES}

11] Porous stainless steel tube (SIKA-R3) manufactured by Newmet Krebsoge, Inc., P.O. Box 68, Terryville, CT 06786.

[2] Lamilloy, manufactured by Allison Gas Turbine Div., General Motor Corp., P.O. Box 420, Indianapolis, Indiana 46206-0420.

[3] Handbook of Heat Transfer, McGraw-Hill, p2$88,1975$. 


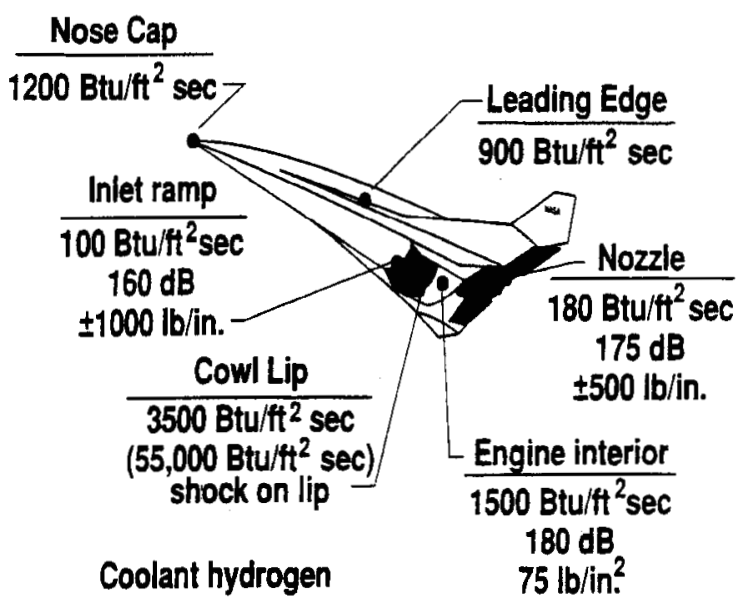

Fig. 1 Estimated heat fluxes on various locations of a hvpersonic vehicle.

\section{TYPICAL HEAT FLUX DISTRIBUTION IN SCRAMJET ENGINE $M=15$}

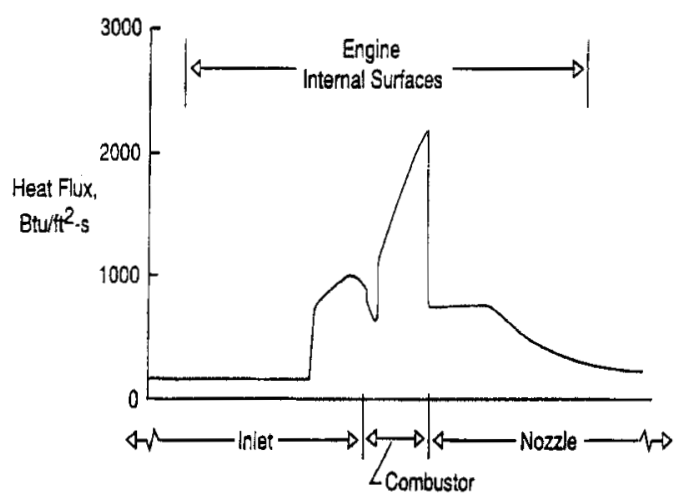

Fig. 2 Typical heat flux distribution in a scram-jet engine.

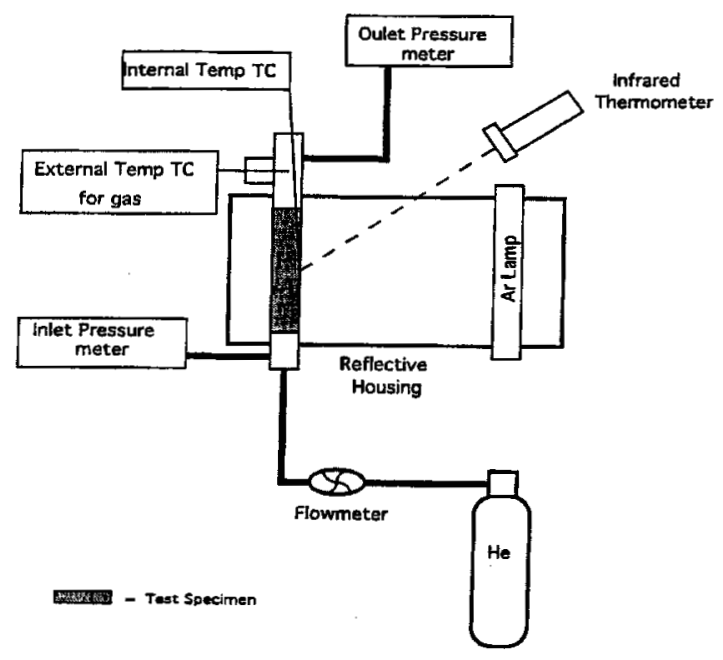

Fig. 3 A block diagram of experimental setup.

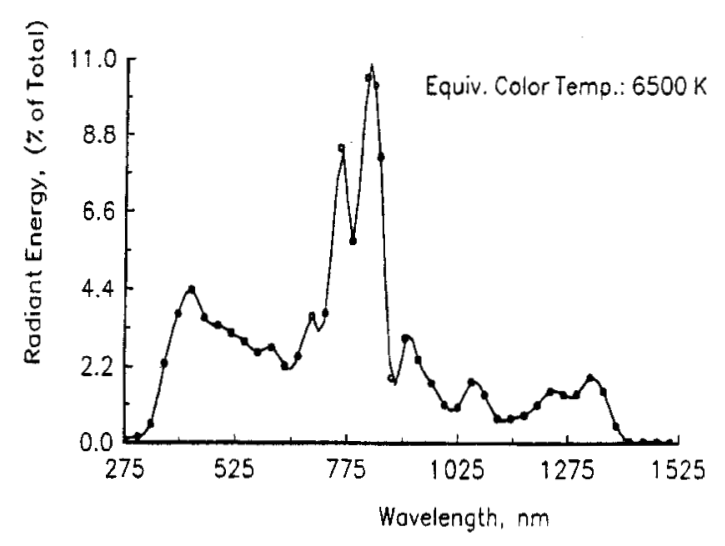

Fig. 4 Spectral energy distribution of argon arc plasma light source.

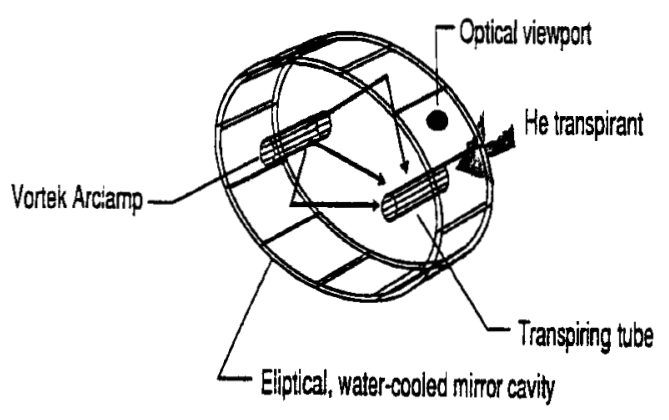

Fig. 5 Elliptical reflector cavity with an argon arc and a specimen, respectively placed on the foci of elliptic reflector.

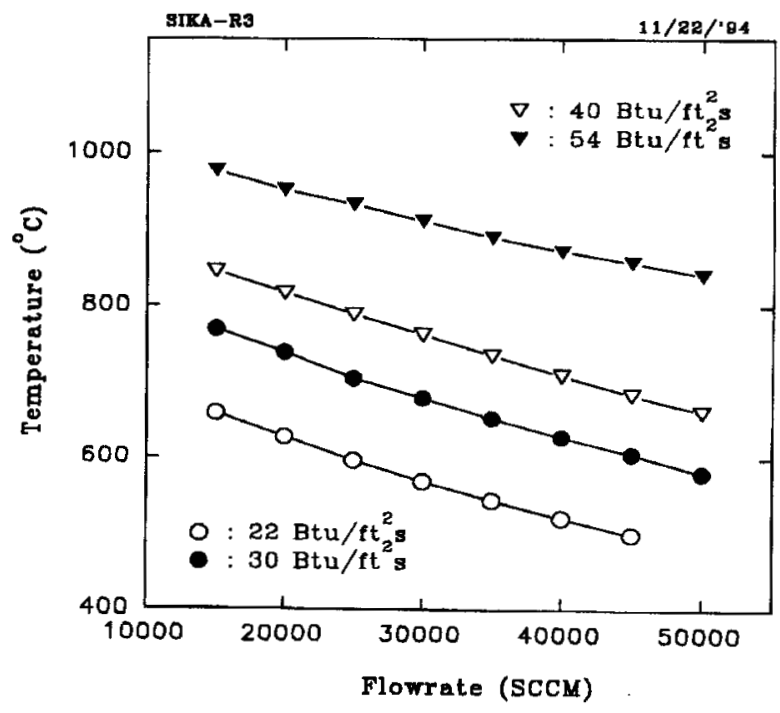

Fig. 6 Outside surface temperatures of a sintered tube (3-micron). 


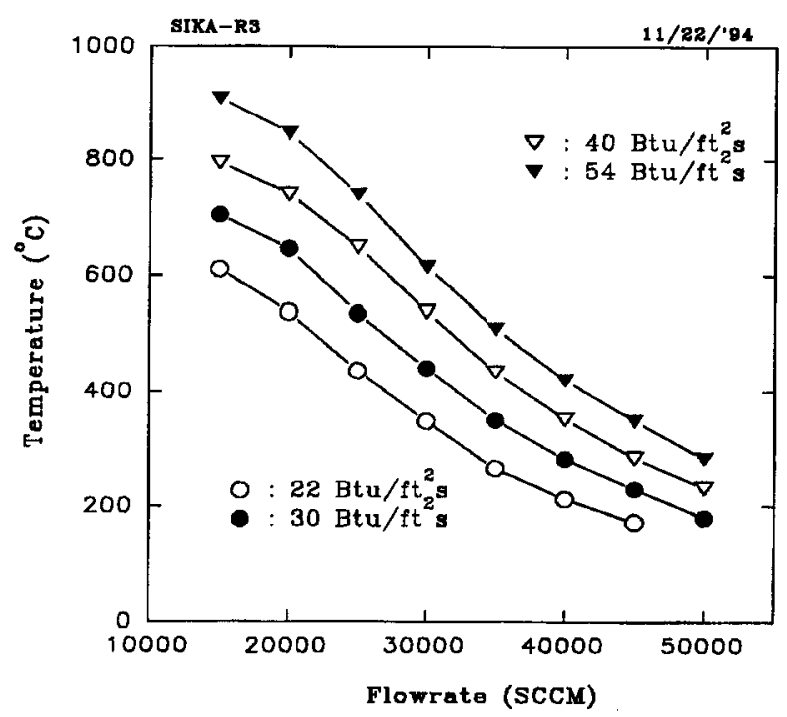

Fig. 7 Inside surface temperatures of a sintered tube (3-micron).

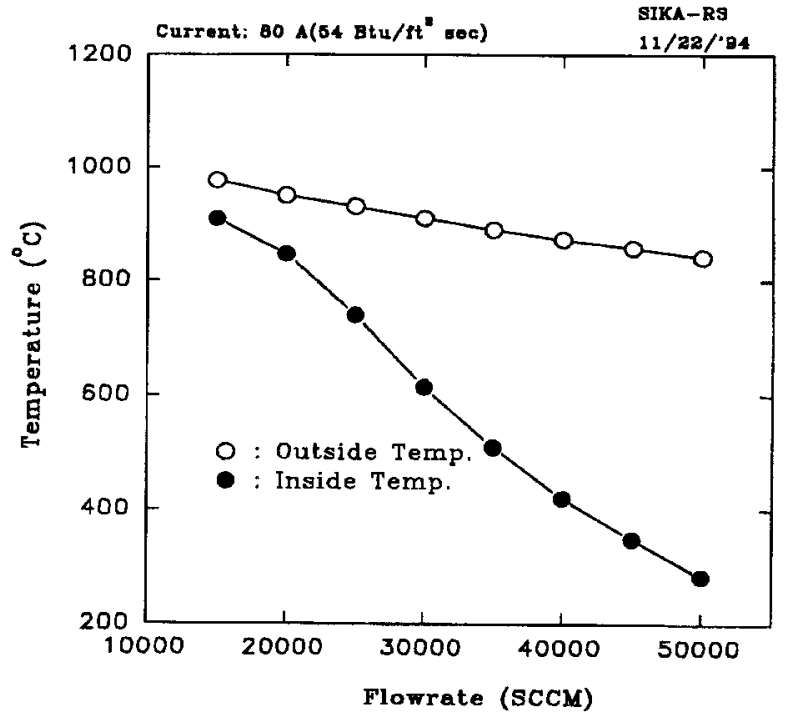

Fig. 8 Temperature variation of inside and outside surfaces.

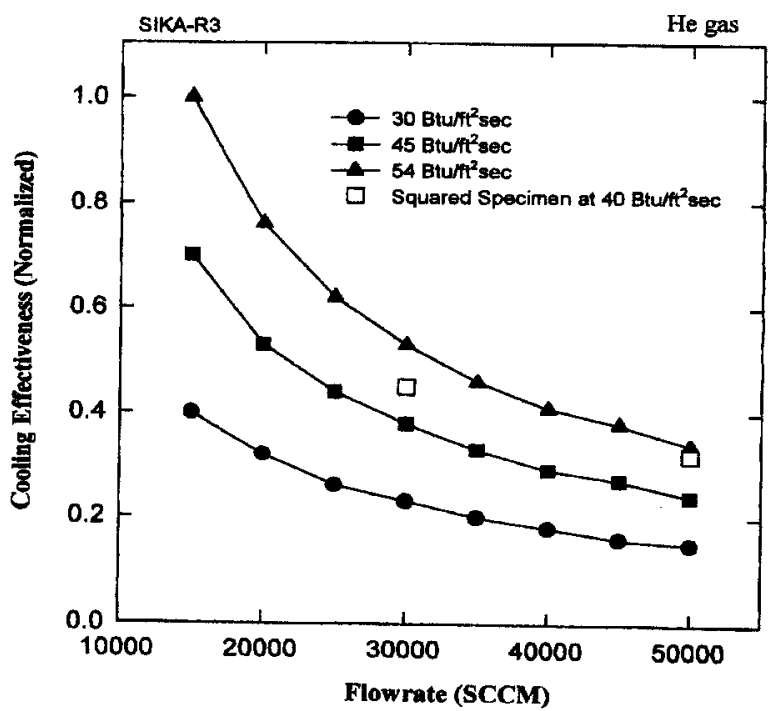

Fig. 9 Transpiration cooling efficiency of a sintered and a multi-layered tubes.

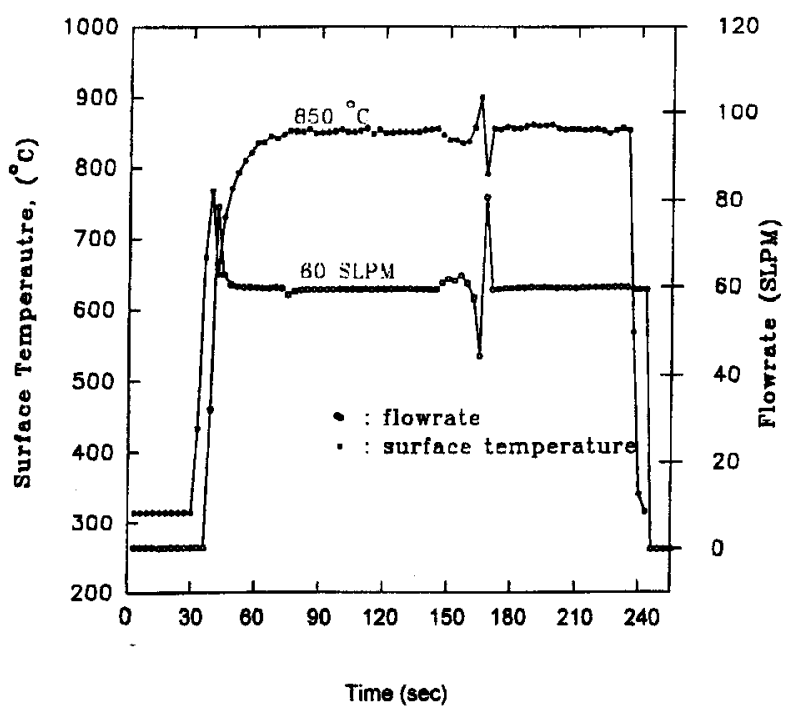

Fig. 10 Surface temperature response to transpirant flow rates.

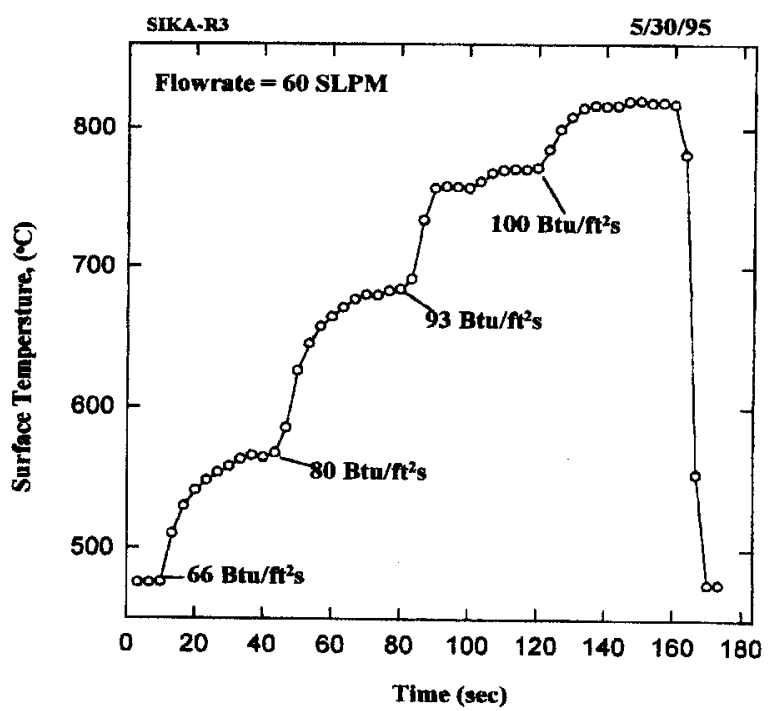

Fig. 11 Surface temperatures corresponding to various heat fluxes at a fixed flowrates.

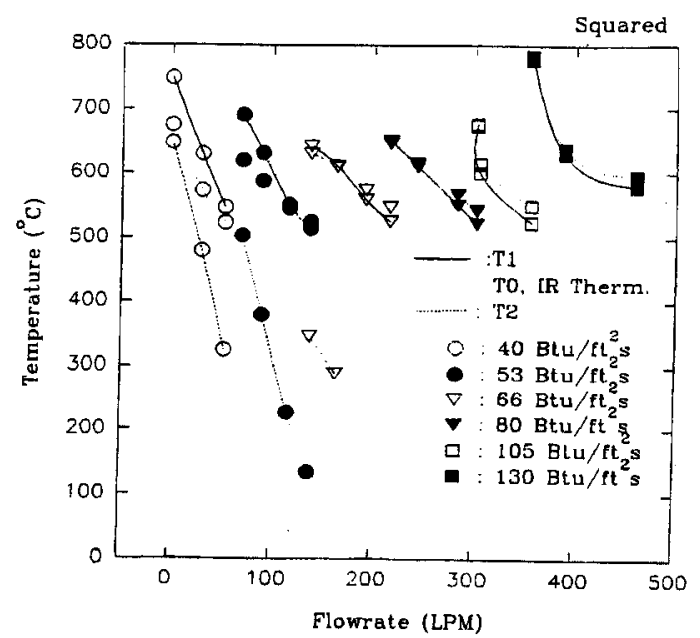

Fig. 12 Surface temperatures of the square cross-section tube with multi-layered and multiperforated holes. 


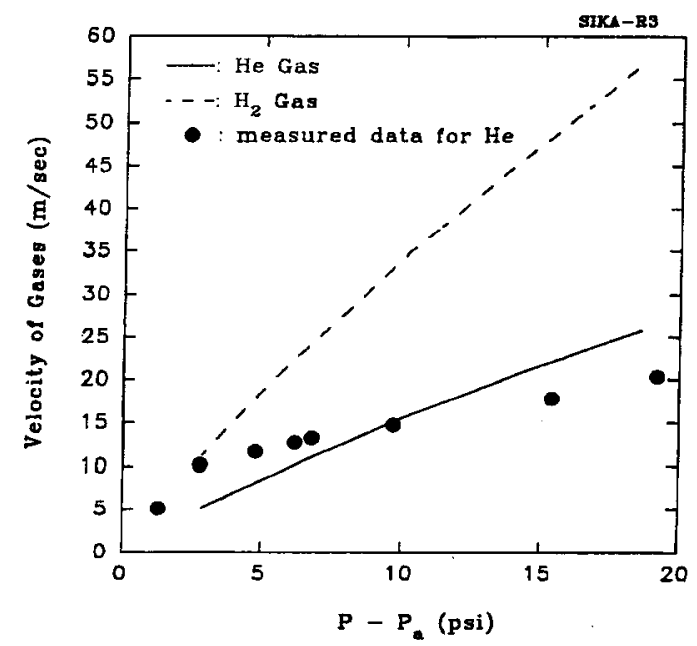

Fig. 13 Velocities of helium and hydrogen gases through a porous material.

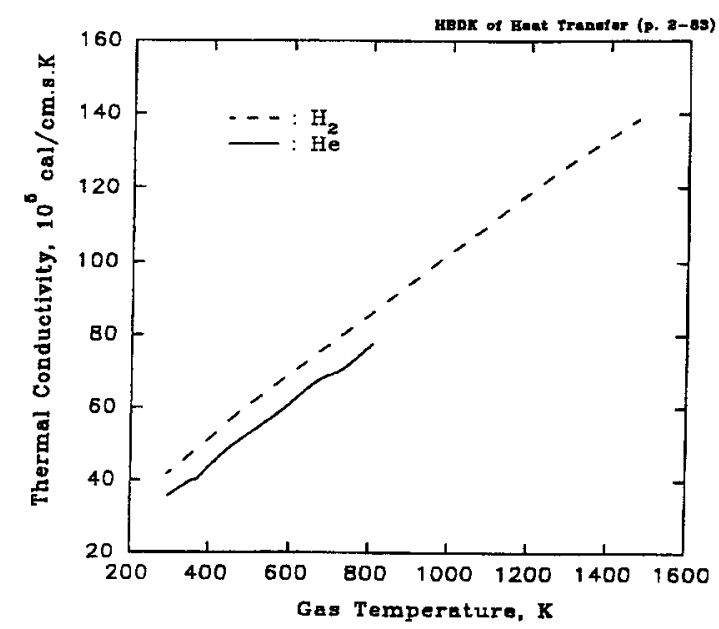

Fig. 14 Thermal conductivities of helium and hydrogen.

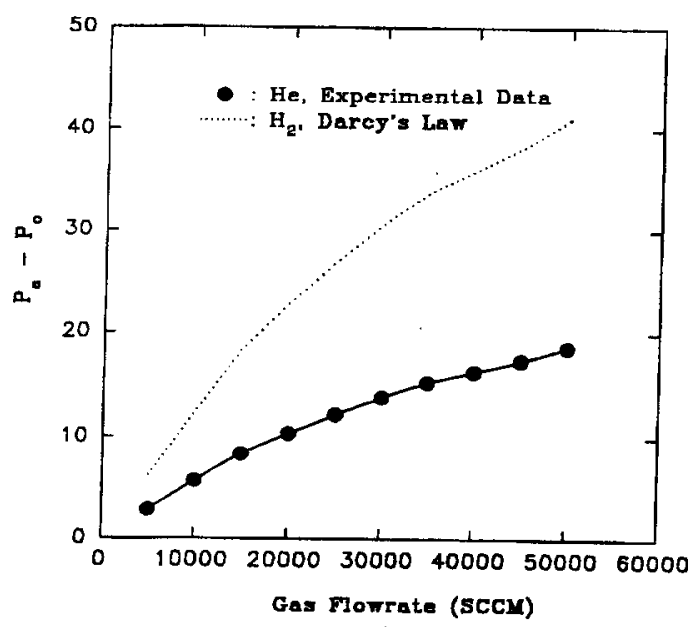

Fig. 15 Gas flowrates vs. pressure drop for helium and hydrogen.

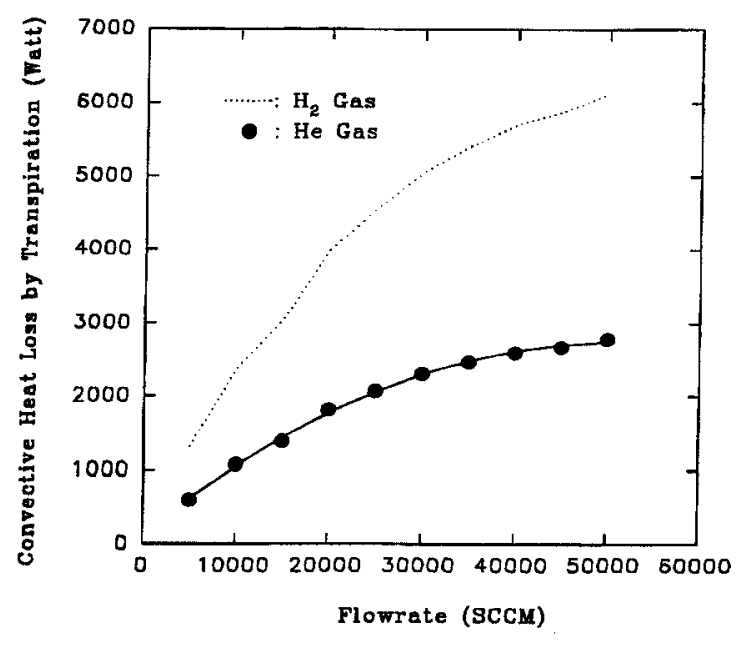

Fig. 16 Convective heat losses due to the flowrates of helium and hydrogen gases through the pores of a tube specimen.

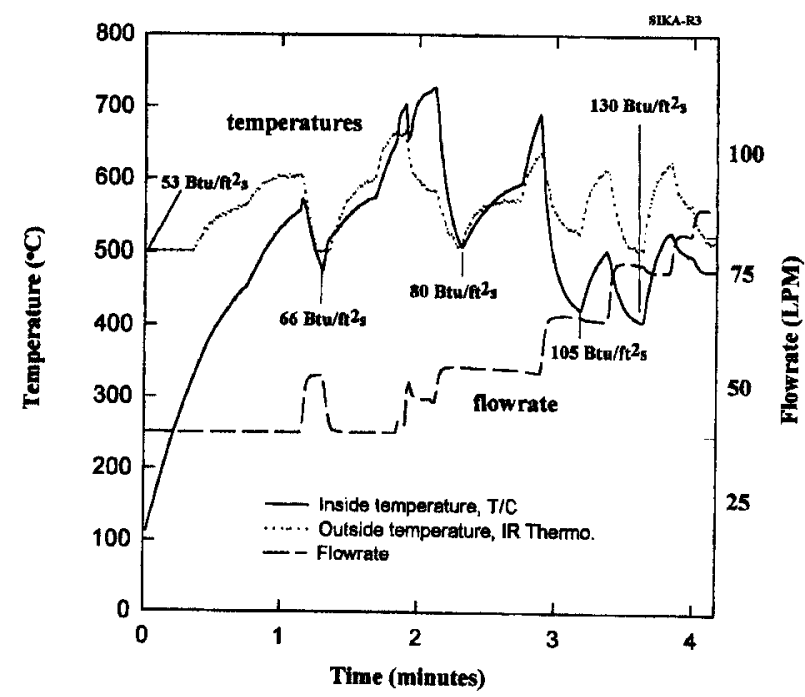

Fig. 17 Temperature responses of a 3-micron sintered tube element with respect to heating loads and helium flow rates.

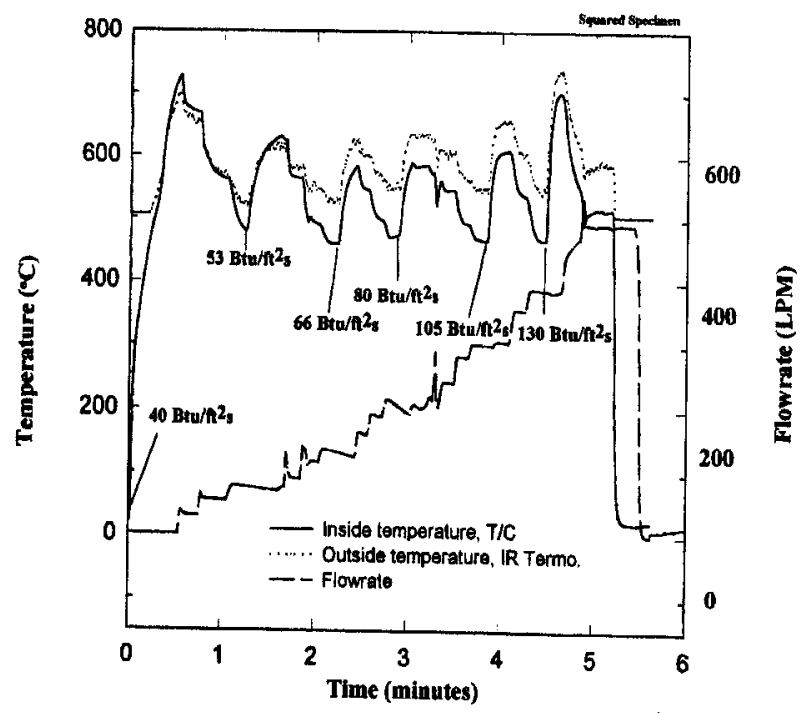

Fig. 18 Temperature responses of a square cross-section tube element with respect to heating loads and helium flow rates. 